\title{
CONTRIBUIÇÕES DA \\ FONÉTICA PARA O ESTUDO \\ DA APRENDIZAGEM DO \\ COMPONENTE \\ SONORO EM L2
}

\author{
CONTRIBUCIONES DE LA FONÉTICA PARA EL ESTUDIO DEL APRENDIZAJE \\ DEL COMPONENTE SONORO EN L2
}

CONTRIBUTIONS OF PHONETICS TO THE STUDY OF L2 SPEECH LEARNING

Rosane Silveira*

Universidade Federal de Santa Catarina

RESUMO: Desde meados dos anos 90, as contribuições da fonética para os estudos da fala em segunda língua (L2) têm aumentado exponencialmente. Esse campo de pesquisa sempre foi fortemente influenciado pelas teorias e hipóteses sobre a aprendizagem de L2, mas, a partir dos anos 90, passou a adotar, também, modelos teóricos voltados exclusivamente para o estudo da fala. Neste artigo, buscamos apontar as principais contribuições da fonética nas pesquisas que buscam compreender como se dá a aprendizagem fonética e fonológica em uma L2. Inicialmente, serão destacadas as principais teorias e metodologias que têm informado os estudos da fala em L2 desde os anos 60. Em seguida, essas influências teóricas e metodológicas serão ilustradas com pesquisas produzidas pelo grupo Fonética e Fonologia Aplicadas à Língua Estrangeira (NUPFALLE). Este breve panorama mostra como a interface fonética-fonologia passou a ser fundamental para o desenvolvimento das pesquisas sobre a aprendizagem do componente sonoro da L2.

PALAVRAS-CHAVE: Fonética. Fonologia. Segunda Língua. Pesquisa.

RESUMEN: Desde mediados de los años 90, las contribuciones de la fonética para los estudios del habla en segunda lengua (L2) han aumentado exponencialmente. Este campo de investigación siempre fue fuertemente influenciado por las teorías e hipótesis sobre el aprendizaje de L2, pero, a partir de los años 90, pasó a adoptarse, también, modelos teóricos dirigidos exclusivamente para el estudio del habla. En este artículo, buscamos apuntar las principales contribuciones de la fonética en las investigaciones que buscan comprender cómo se da el aprendizaje fonético y fonológico en una L2. En primer lugar, se destacarán las principales teorías y metodologías que han informado los estudios del habla en L2 desde los años 60. A continuación, estas influencias teóricas y metodológicas serán ilustradas con investigaciones producidas por el grupo Fonética y Fonología Aplicadas a la Lengua Extranjera

\footnotetext{
* Professora do Departamento de Língua e Literatura Estrangeiras da UFSC e do Programa de Pós-Graduação em Inglês. Bolsista Produtividade do CNPq e coordenadora do grupo de pesquisa Núcleo de Fonética e Fonologia Aplicadas à Língua Estrangeira. E-mail: rosanesilveira@hotmail.com.
} 
(NUPFALLE). Este breve panorama muestra cómo la interfaz fonética-fonología pasó a ser fundamental para el desarrollo de las investigaciones sobre el aprendizaje del componente sonoro de la L2.

PALABRAS CLAVE: Fonética. Fonología. Segunda Lengua. Investigación.

ABSTRACT: Since the mid-90s, the contributions of phonetics to second language (L2) speech studies have increased exponentially. This field of research has always been strongly influenced by theories and hypotheses about L2 learning, but since the mid-90s, it has also adopted theoretical models focused exclusively on the study of speech. In this article, we seek to highlight the main contributions of phonetics in studies that seek to understand how phonetic-phonological learning occurs in an L2. Initially, the main theories and methodologies that have informed L2 speech studies since the 1960s are highlighted. These theoretical and methodological influences are illustrated with research conducted in Brazil, more specifically research from the group Phonetics and Phonology Applied to Foreign Language (NUPFALLE). This brief overview shows how the phonetics-phonology interface has become

KEYWORDS: Phonetics. Phonology. Second Language. Research.

\section{INTRODUÇÃO}

O estudo da fala em uma língua diferente da língua materna (doravante L2) tem como ponto de partida algumas perguntas centrais ligadas à aprendizagem fonética e fonológica: somos capazes de incorporar novos sons depois que a gramática fonológica da L1 já está constituída? Como lidamos com as diferenças fonotáticas entre a L1 e a L2? Como percebemos/produzimos os fonemas/alofones da L2? Convivemos com um sistema único para a L1 e para a L2? Ou há um sistema para cada língua? São inúmeros os estudos que buscam, de alguma forma, responder essas perguntas, como podemos constatar em trabalhos que resenham esse tipo de pesquisa (ZIMMER; SILVEIRA; ALVES, 2009; ALVES, 2015; SILVEIRA et al., 2017).

Nos últimos anos, o campo da fonética tem contribuído imensamente para os estudos da fala em L2. Neste artigo, buscamos apontar as principais contribuições da fonética nas pesquisas que buscam compreender como se dá a aprendizagem fonética e fonológica em uma L2. Para atingir esse objetivo, iniciaremos destacando as principais teorias e metodologias que têm informado os estudos da fala em L2 desde os anos 60, dando destaque para pesquisas produzidas no Brasil, mais especificamente pelo grupo Fonética e Fonologia Aplicadas à Língua Estrangeira (NUPFALLE), grupo de pesquisa criado na Universidade Federal de Santa Catarina e cadastrado no CNPq desde 2004. O NUPFFALE é constituído de professores e alunos de Pós-Graduação e de Iniciação Científica de várias instituições brasileiras, envolvidos em projetos de pesquisa em fonética e fonologia aplicada à língua estrangeira. Por fim, destacaremos de que forma a fonética tem auxiliado na investigação da fala em L2.

\section{TEORIAS E METODOLOGIAS PRESENTES NA PESQUISA SOBRE A FALA EM L2}

As pesquisas sobre a fala em L2 têm como foco principal examinar a percepção e/ou a produção dos sons (segmentos vocálicos ou consonantais) ou de aspectos suprassegmentais da L2 (ex.: acento lexical, entoação, acento tonal). Uma etapa importante nesses estudos envolve a comparação dos sistemas sonoros de duas ou mais línguas e a descrição de problemas de aprendizagem. Alguns estudos também buscam refletir sobre o ensino dos componentes fonéticos e fonológicos da L2, tendo como objetivo ampliar a inteligibilidade da fala do aprendiz de L2. Por exemplo, estudos que investigam o desenvolvimento da fala em língua inglesa por aprendizes brasileiros podem buscar compreender como o sistema consonantal da L2 é construído pelo aprendiz. Para isso, uma primeira etapa é comparar os sistemas consonantais do português brasileiro (PB) e do inglês. Conforme podemos observar no Quadro 1, o PB possui 19 fonemas consonantais (CRISTÓFARO-SILVA, 2001), ao passo que o inglês possui 24 (CELCE-MURCIA et al., 2010). Diante desse cenário, pode-se prever que aprendizes brasileiros poderão ter dificuldades em adquirir sons que não existem em sua língua materna (/ð/ e / $/$ /), bem como compreender o status de fonema na L2 para sons que são alofones em sua língua materna (ex.: /dz/ e / $\mathrm{t} /$ ). Além disso, terão dificuldade com fonemas que existem em sua L1, porém ocupando posições fonotáticas diferentes na L2, como um /£/ em início de palavra ou /p t k/ em coda silábica. 
Consoantes do PB

\begin{tabular}{|c|c|c|c|}
\hline$/ \mathrm{v} /$ & $/ \mathrm{m} /$ & $/ \mathfrak{v} /$ & $/ \Gamma /$ \\
\hline$/ \mathrm{b} /$ & $/ \mathrm{n} /$ & $/ \mathrm{b} /$ & $|z|$ \\
\hline $\mid t /$ & $/ \mathrm{n} /$ & $/ \mathrm{t} /$ & $/ \mathrm{h} /$ \\
\hline$/ \mathrm{d} /$ & $/ 1 /$ & $/ \mathrm{d} /$ & $/ \mathrm{d} \mathrm{z} /$ \\
\hline$/ \mathrm{k} /$ & $|K|$ & $/ \mathrm{k} /$ & $/ \mathrm{t} /$ \\
\hline$/ \mathrm{g} /$ & $|r|$ & $\mid \mathrm{g} /$ & $/ \mathrm{m} /$ \\
\hline $\mid \mathrm{f} /$ & $/ \mathrm{R} /$ & $/ \mathrm{f} /$ & $/ \mathrm{n} /$ \\
\hline$/ \mathrm{v} /$ & & $/ \mathrm{v} /$ & $/ \mathrm{n} /$ \\
\hline $\mid \mathrm{s} /$ & & /ð/ & /1/ \\
\hline$|z|$ & & $\mid \theta /$ & $/ \mathrm{J} /$ \\
\hline$/ \Gamma /$ & & $/ \mathrm{s} /$ & $/ \mathrm{w} /$ \\
\hline$|z|$ & & $|\mathrm{z}|$ & $/ \mathrm{v} /$ \\
\hline
\end{tabular}

Quadro 1: Comparação entre os fonemas consonantais do português brasileiro e do inglês

Fonte: produzido pela autora

Para compreender melhor de que forma a fonética passou a desempenhar um papel central nas pesquisas sobre a fala em L2, é interessante traçar um breve panorama das principais influências teóricas e metodológicas nos estudos da L2 a partir dos anos 60, época em que os estudos no campo da L2 começam a ser disseminados (MITCHELL; MYLES, 2004). Veremos que as pesquisas da fala em L2 são fortemente influenciados por teorias ou hipóteses sobre a aprendizagem de uma L2, e que algumas dessas perspectivas teóricas demandam uma abordagem multidisciplinar para investigar a aprendizagem de uma L2, incluindo a aprendizagem do componente sonoro.

Entre os anos 60 e 90, a principal discussão permeando a área da fala em L2 girava em torno de um questionamento: o que influencia a pronúncia em L2: a L1 do aprendiz ou princípios universais da linguagem? Esse debate era sustentado, inicialmente, pela Hipótese da Análise Contrastiva (LADO, 1957), cuja principal premissa é a de que a fonologia da L1 influencia a fonologia da L2. O desdobramento metodológico dessa premissa é que os estudos sobre a fala em L2 buscavam identificar diferenças no inventário fonêmico das duas línguas, detectar ausências de fonemas da L2 na L1 e prever substituições a serem feitas pelo aprendiz da L2, com base na transferência de fonemas da L1 para a L2.

As pesquisas desse período tinham como foco generalizações feitas com base nas intuições do pesquisador sobre as diferenças entre a L1 e a L2, ou, posteriormente, a análise de produção da fala em L2. Além da abordagem intuitiva, as pesquisas se valiam da análise de oitiva para a transcrição fonética e a classificação das produções orais dos informantes.

Nos anos 70, o conceito de interlíngua ganha força (CORDER, 1971; SELINKER, 1972), defendendo a ideia de que a língua do aprendiz de L2 não é idêntica nem à L1 nem à L2. Portanto, a interlíngua consiste em "um sistema em si mesmo com estrutura própria" (GASS; SLINKER, 2008, p. 14 ${ }^{1}$, tradução da autora), contendo elementos da L1, da L2 e novas formas. A metodologia que informa os estudos da fala influenciados pelo princípio da interlíngua consiste em ouvir as produções dos informantes, identificar tipos de erros de pronúncia e suas origens (L1 ou universais), bem como detectar se a interlíngua continua em desenvolvimento ou se 'fossilizou', ou seja, se a aprendizagem cessou (GASS; SLINKER, 2008).

1 "[...] a system of its own with its own structure [...]" (GASS; SLINKER, 2008, p. 14). 
Já no final dos anos 70, surge a Markedness Differencial Hypothesis (ECKMAN, 1977), advogando que vários tipos de universais linguísticos influenciam a interlíngua e que aspectos mais marcados (i.e., menos frequentes nas línguas) levam a maior dificuldade de aquisição. O número de estudos testando essa hipótese é bastante significativo no campo da fala em L2, e esses estudos adotam a metodologia de prever aspectos mais ou menos marcados na L1 e na L2 e verificar se os que são mais marcados na L2 causam mais dificuldade de aprendizagem, examinando auditivamente dados de fala dos informantes.

Em meados dos anos 80, surge o Ontogeny Philogeny Model (MAJOR, 1986), o qual propõe que, inicialmente, a L1 influencia mais a aprendizagem do componente sonoro da L2; posteriormente, princípios universais passam a ter um papel central. No entanto, à medida que o aprendiz se torna mais proficiente, os dois tipos de influência diminuem. A metodologia adotada por estudos que testam o modelo de Major (1986) é muito parecida com aquela adotada nos anos 60 e 70, mas uma prática fundamental é comparar diferentes níveis de proficiência, especialmente em estudos com design transversal, para identificar erros de produção e classificar como erros resultantes de transferência da L1 ou de processos naturais de desenvolvimento da fala (universais).

Nos anos 90, podemos notar uma grande mudança de perspectivas teóricas e metodológicas nos estudos da fala em L2. Tal mudança é impulsionada pelo surgimento de vários modelos teóricos que apresentam forte influência da psicologia cognitiva e a preferência por abordagens experimentais. O mais influente desses modelos é o Speech Learning Model (FLEGE, 1995), que tem como premissa central investigar como a percepção influencia a produção dos sons da L2. Pesquisas que adotam esse modelo buscam compreender a importância das diferenças articulatórias e do status do(s) segmento(s) (fonético ou fonológico) que estão sendo investigados. A metodologia, então, precisa incluir a análise de dados acústicos tanto da L1 quanto da L2 e, preferencialmente, correlacionar o desempenho de aprendizes ou usuários da L2 em testes de percepção e produção do componente sonoro investigado.

Outro modelo bastante influente a partir de meados dos anos 90 foi o PAM - Perception Assimiliation Model, proposto por Best (1995) e revisado para os estudos em L2 por Best and Tyler (2007) - PAML2. Esse modelo é bastante utilizado pelos estudos que investigam essencialmente a percepção dos sons da L2. A metodologia também é de cunho experimental e tende a incluir uma variedade maior de testes de percepção. A premissa central desses modelos é a de que a habilidade para discriminar pares de fonemas depende da forma como cada um desses fonemas é assimilado pelo aprendiz. Na aprendizagem de uma L2, um contraste fonêmico cujos fonemas são igualmente assimilados a uma única categoria fonológica da L1, prevê-se que o aprendiz terá dificuldades para discriminar entre esses sons. Por exemplo, um brasileiro que percebe as vogais /I/ e /i/ do inglês como sendo equivalentes à vogal /i/ do PB terá dificuldades para discriminar entre esses dois fonemas da língua inglesa.

É graças a forte influência da perspectiva experimental dos anos 90 que passamos a observar a utilização de procedimentos de análise da fonética nas pesquisas da fala em L2, com especial destaque para a fonética acústica e perceptual. A metodologia envolve coleta de dados mais ampla e comparação efetiva entre dados de fala da L1 e da L2. Neste momento, a sociolinguística também passa a influenciar metodologicamente as pesquisas da fala em L2, com a inserção de variáveis que buscam compreender melhor as diferenças individuais e do contexto de aprendizagem e/ou exposição à L2. A análise dos dados pode incluir o julgamento de ouvintes especializados, mas precisa, necessariamente, ser acompanhada de análise acústica, quer seja para a elaboração dos estímulos dos testes de percepção, quer seja para a análise dos dados de produção. A comparação entre dados de monolíngues e bilíngues é fundamental dentro desta perspectiva.

Recentemente, a área de estudos sobre a fala em L2 tem sido influenciada pela Teoria da Complexidade, mais especificamente, pela proposta dos Sistemas Dinâmicos e Adaptativos Complexos (LARSEN-FREEMAN; CAMERON, 2008; DE BOT, 2017), a qual preconiza que a aprendizagem de uma L2 emerge da interação entre vários fatores (e.g., individuais, cognitivos, contextuais e linguístico), que tal aprendizagem é não-linear, e que há uma trajetória única para cada aprendiz. Metodologicamente, essa perspectiva teórica requer a continuidade da perspectiva experimental, mas é fundamental analisar e considerar a variabilidade e a gradiência dos dados, bem como observar relações entre dados linguísticos e não linguísticos e priorizar a análise de dados longitudinais e intra-indivíduo. 
Cabe notar que a valorização dos percursos individuais dos aprendizes de L2 implica grandes mudanças metodológicas que ainda estão sendo construídas pelos pesquisadores da área (LOWIE, 2017). Um ponto crucial nessa mudança é aceitar que o sistema linguístico de um falante de L2 não deve ser equiparado ao sistema de um falante monolíngue. Portanto, a comparação com dados de falantes monolíngues não deve, necessariamente, ser vista como a forma ideal para analisar dados de percepção e produção da fala em L2. Consequentemente, passamos a ter estudos que se voltam para a descrição do sistema do aprendiz e do grau de inteligibilidade da linguagem produzida pelo aprendiz, segundo a perspectiva de outros aprendizes com línguas maternas diversas e, eventualmente, segundo a perspectiva de falantes nativos da L2 sendo investigada.

Estudos informados teoricamente pelos modelos dos anos 90 (ex.: SLM, PAM, PAML2) e pela teoria dos Sistemas Dinâmicos Adaptativos Complexos demandam uma forte interface entre a fonética e a fonologia. Frequentemente, os estudos que demandam tal interface têm sido identificados com a perspectiva da Fonologia de Laboratório (BECKMAN; KINGSTON, 1990; PIERRHUMBER, BECKMAN; LADD, 2000), termo cunhado por Pierrhumbert (1987) para descrever uma perspectiva científica que utiliza uma abordagem multidisciplinar para investigar como a língua falada é estruturada, aprendida e utilizada (COHN; FOUGERON; HUFFMAN, 2012). A perspectiva da Fonologia de Laboratório requer a incorporação de metodologias experimentais de outras áreas (fonética, aquisição, sociolinguística, psicolinguística) para compreender a fala humana (COHN; FOUGERON; HUFFMAN, 2012).

Este breve panorama mostra de que forma a pesquisa sobre a aprendizagem da fala em L2 foi se constituindo desde os anos 60 até o momento, e como a interface fonética-fonologia passou a ser fundamental para o desenvolvimento dessa área. Na próxima seção, ilustraremos essas mudanças teóricas e metodológicas no âmbito do grupo de pesquisa NUPFFALE, formado por pesquisadores brasileiros interessados em investigar a fala em L2.

\section{BREVE PANORAMA DAS PESQUISAS DO GRUPO NUPFFALE}

Nesta seção, buscamos exemplificar a forma como as perspectivas teóricas descritas na seção anterior podem ser observadas nas pesquisas produzidas no âmbito do grupo NUPFFALE.

Os primeiros estudos do grupo de pesquisa foram orientados pela profa. Barbara Oughton Bapatista, em meados dos anos 90. Nas primeiras dissertações de mestrado, podemos observar uma forte Influência dos trabalhos de Eckman (1977) e Major (1986). Por exemplo, o Quadro 2 apresenta os trabalhos de Rebello (1997) e Silva Filho (1998), os quais foram claramente inspirados na Markedness Theory, tendo como foco principal a análise da produção de padrões silábicos da L2 em termos de fatores linguísticos como sonoridade, ponto e modo de articulação e contexto fonológico.

\begin{tabular}{|c|c|c|}
\hline Autor & Nível & Título \\
\hline Rebello (1997) & Mestrado & The acquisition of English initial /s/ clusters by Brazilian learners. \\
\hline Silva Filho (1998) & Mestrado & The production of English syllable-final consonants by Brazilian learners \\
\hline $\begin{array}{c}\text { Cornelian Jr. } \\
\quad(2003)\end{array}$ & Mestrado & Brazilian learners' production of /s/ clusters: Phonological structure and environment. \\
\hline
\end{tabular}

Quadro 2: Exemplos de pesquisas conduzidas nos anos 90

Fonte: produzido pela autora

Cabe destacar que esses estudos investigavam exclusivamente dados de produção da fala e que a análise de oitiva era utilizada para transcrever foneticamente a produção dos aprendizes. A mesma perspectiva teórica e metodológica foi adotada, de forma integral 
ou parcial, por outras dissertações e teses produzidas pelo grupo (ex., BETTONI-TECHIO, 2005; DELATORRE, 2006; SILVEIRA, 2004).

Conforme demonstra o Quadro 3, nos estudos realizados entre os anos 2000-2005, já podemos observar a influência do SLM de Flege (1995), com a discussão sobre a relação entre percepção e produção da fala em L2.

Autor

Nível

\begin{tabular}{ccc}
\hline Koerich (2002) & Doutorado & Perception and production of word-final vowel epenthesis by Brazilian EFL students \\
Silveira (2004) & Doutorado & The influence of instruction on the perception and production of English word-final consonants \\
Kluge (2004) & Mestrado & Perception and production of English syllable-final nasals by Brazilian learners \\
\hline
\end{tabular}

Quadro 3: Exemplos de pesquisas influenciadas pela fonética perceptual (início dos anos 2000)

$$
\text { Fonte: produzido pela autora }
$$

Koerich (2002) produziu o primeiro trabalho do grupo NUPFFALE no campo da percepção da fala em L2, bem como o primeiro que busca a interface entre fonética e fonologia. De fato, ao olharmos a metodologia da pesquisa, é possível observar que os dados de produção foram coletados e analisados seguindo a tradição dos estudos das décadas anteriores, ou seja, a análise era baseada na transcrição fonética feita por especialistas e na categorização das produções. Já nos dados de percepção, aparece a utilização da metodologia da fonética perceptual. Procedimentos metodológico semelhantes são utilizados nos estudos de Silveira (2004) e Kluge (2004).

Há que se ressaltar que a fundadora do grupo NUPFFALE já buscava a interface entre fonética e fonologia em sua própria pesquisa de doutorado (BAPTISTA, 1992), a qual investigou a aquisição das vogais da língua inglesa por brasileiros e foi conduzida no laboratório de fonética da University of California at Los Angeles

A pesquisa de Baptista (1992) adota uma perspectiva metodológica bastante diferente das pesquisas orientadas por ela no início de sua atuação no Programa de Pós-Graduação em Inglês. A metodologia adotada é claramente informada pela fonética acústica, uma vez que a análise dos dados é feita com base nos valores dos formantes das vogais. Além disso, a coleta dos dados adota uma perspectiva longitudinal, o que permite discutir o papel da proficiência na construção da gramática fonológica da L2, como proposto pelo modelo de Major (1986).

A presença marcante da fonética acústica pode ser observada em duas pesquisas de mestrado conduzidas em meados dos anos 2000. Como mostra o Quadro 4, Cohen (2004) conduziu o primeiro estudo investigando os valores de VOT (voice onset time), comparando dados da L1 e da L2 de brasileiros, aprendizes de inglês. Ribeiro (2006) conduziu a primeira pesquisa investigando os correlatos acústicos (duração, frequência fundamental, e pico de intensidade) do acento lexical em dados do inglês e do português brasileiro.
Autor
Nível
Título

Cohen (2004) Mestrado The VOT dimension: A bi-directional experiment with English and Brazilian-Portuguese stops
Acoustic correlates of word-stress production in the connected speech of American English and
Brazilian Portuguese speakers.

Quadro 4: Exemplos de pesquisas com forte influência da fonética acústica (meados dos anos 2000)

Fonte: produzido pela autora 
Como podemos observar no Quadro 5, entre 2006 e 2010, os pesquisadores do NUPFFALE passaram a explorar cada vez mais as alternativas metodológicas da fonética acústica e perceptual para investigar a percepção e a produção da fala em L2 por aprendizes brasileiros. A tese de Rauber (2006) demonstra uma clara ênfase na metodologia da fonética acústica e perceptual para o estudo de vogais.

\begin{tabular}{|c|c|c|}
\hline Autor & Nível & Título \\
\hline Rauber (2006) & Doutorado & Perception and production of English vowels by Brazilian EFL speakers \\
\hline Nobre-Oliveria (2007) & Doutorado & $\begin{array}{l}\text { The effect of perceptual training on the learning of English vowels by Brazilian } \\
\text { Portuguese speakers }\end{array}$ \\
\hline Kluge (2009) & Doutorado & $\begin{array}{l}\text { Brazilian EFL learners' identification of word-final } / \mathrm{m}-\mathrm{n} / \mathrm{:} \text { native/nonnative } \\
\text { realizations and effect of visual cues }\end{array}$ \\
\hline Bion (2007) & Mestrado & $\begin{array}{l}\text { The role of listeners' dialect in the perception of foreign } \\
\text { accented vowels }\end{array}$ \\
\hline Baratieri (2006) & Mestrado & $\begin{array}{l}\text { Production of /l/ in the English coda by Brazilian EFL learners: An acoustic- } \\
\text { articulatory analysis }\end{array}$ \\
\hline
\end{tabular}

Quadro 5: Exemplos de pesquisas com forte influência da fonética acústica e perceptual (2006-2010)

Fonte: produzido pela autora

Ainda na segunda metade dos anos 2000, como mostra o Quadro 5, os estudos voltados para a percepção da fala em L2 passaram, também, a utilizar técnicas de manipulação de pistas acústicas para melhor compreender a percepção da fala em L2. Nobre-Oliveira (2007), por exemplo, utilizou a manipulação de pistas acústicas (valores de F1 e F2, bem como duração das vogais) para verificar o efeito do treinamento perceptual (baseado em estímulos produzidos naturalmente e estímulos acusticamente manipulados) na aprendizagem de vogais da língua inglesa por aprendizes brasileiros.

A noção de que a construção do sistema fonético-fonológico se dá com base em múltiplos fatores fica evidente, também, na tese de Kluge (2009), a qual destaca o efeito da informação acústica e visual na percepção de codas consonantais nasais da língua inglesa por brasileiros. Além disso, a dissertação de Bion (2007) utiliza a análise acústica de vogais produzidas por falantes de inglês (nativos e não nativos) de diferentes nacionalidades com o intuito de explicar como essas vogais são percebidas por falantes nativos do inglês.

O Quadro 5 também ilustra que, no campo da produção da fala em L2, começam a aparecer trabalhos que investigam o papel do detalhe acústico na produção de consoantes. Baratieri (2006) analisa os dados de produção acusticamente e destaca a gradiência da líquida produzida por brasileiros, revelando diferentes estágios de aquisição para a consoante líquida.

Atualmente, as pesquisas do grupo NUPFFALE investigam o papel do detalhe fonético e do tipo de input que o aprendiz de L2 recebe, em especial quando pensamos no aprendiz da língua inglesa, utilizada como língua de comunicação internacional por falantes de inúmeras nacionalidades. As pesquisas têm, cada vez mais, visto a aprendizagem do componente sonoro em L2 como um fenômeno complexo no qual o aprendiz está exposto a dados com diferentes características acústicas e articulatórias e deve se comunicar com ouvintes que não são necessariamente falantes nativos da língua que está sendo utilizada para comunicação.

Como pode ser observado no Quadro 6, a tese de Delatorre (2017) discute a natureza complexa da aprendizagem sonora em L2, voltando o olhar para o detalhe fonético de produções do -ed de diferentes grupos de falantes do inglês e para a inteligibilidade dessas produções para aprendizes brasileiros. 
Autor Nível Título

\begin{tabular}{|c|c|c|}
\hline Delatorre (2017) & Doutorado & Intelligibility of English verbs ending in -ed for Brazilian learners of English listeners \\
\hline $\begin{array}{l}\text { Passarella dos Reis } \\
\text { (2017) }\end{array}$ & Doutorado & $\begin{array}{c}\text { "What do you mean?": Nuclear stress in English as an international language: Uses and } \\
\text { interpretations" }\end{array}$ \\
\hline Gonçalves (2017) & Doutorado & The orthographic signature in second language speech acquisition and processing. \\
\hline
\end{tabular}

Quadro 6: Exemplo de pesquisas a partir de 2010

Fonte: produzido pela autora

Já a tese de Passarella-dos Reis (2017) é a primeira a investigar as características acústicas do acento nuclear de enunciados em língua inglesa produzidos por brasileiros e a inteligibilidade desses enunciados, com base no julgamento de ouvintes em situação de interação comunicativa. Por fim, o Quadro 6 mostra que, no campo do processamento da fala em L2, a tese de Gonçalves (2017) investigou de que forma a ortografia é acionada quando aprendemos sons de uma L2, examinando a aprendizagem de um léxico artificial.

\section{CONCLUSÃO}

Esse breve panorama de pesquisas conduzidas pelo grupo NUPFFALE mostra que, no campo da fala em L2, os trabalhos desenvolvidos desde o início dos anos 2000 constantemente buscam a interface entre fonética e fonologia. As pesquisas estão alinhadas à perspectiva da Fonologia de Laboratório, demonstrando a coexistência de "padrões sutis, gradientes e variáveis (i.e., fonéticos) e padrões completos e categóricos (i.e., fonológicos) (SCOBBIE 2007, p. 18).

A utilização de metodologias do campo da fonética auxilia na análise dos dados de fala e na compreensão do complexo fenômeno da construção da gramática sonora de uma L2. Como explica SCOBBI, (2007, p. 30), as categorias discretas da fonologia nem sempre ajudam a entender o desenvolvimento fonológico em L2. Além disso, as intuições do falante ou os julgamentos do pesquisador também estão sujeitos à variação e são influenciados pela ortografia e atitudes sociolinguísticas.

Para as pesquisas da fala em L2 que buscam uma interface com o ensino de línguas (SILVEIRA, 2004; ALVES, 2004; NOBREOLIVEIRA, 2007), a inclusão de metodologias da fonética foi fundamental para compreender que o sotaque não é um problema porque a variação é a norma e não a exceção no desenvolvimento da fala. Ao partir desse princípio, fica claro que os aspectos fonológicos devem ser priorizados no ensino de L2. O detalhe fonético pode ser abordado no ensino de L2 porque pode aumentar a inteligibilidade da fala e a compreensão oral, mas não é fundamental, pois não se tem como objetivo eliminar o sotaque do aprendiz de L2, nem tampouco emular a fala ou a percepção de um falante nativo idealizado. A análise fonética também permite novos insights sobre as características acústicas dos dados de produção utilizados como estímulo nas pesquisas e aos quais o aprendiz de L2 está exposto.

Como ressalta Ladefoged (1972), há muitos tipos de interface entre fonética e fonologia, dependendo dos objetivos da pesquisa e de seus pressupostos. Os estudos da fala em L2 claramente buscam um tipo de interface que auxilia na resposta de perguntas de pesquisa e que gera novas perguntas a serem investigadas. Como ressalta Davidson (2011, p. 126, tradução da autora),

O estudo da [... fala em L2 tem se beneficiado de pesquisas de vários subcampos da linguística. Insights advindos da fonologia, da fonética acústica, perceptual e articulatória têm influenciado nossa compreensão sobre como falantes aprendem a produzir uma língua estrangeira. ${ }^{2}$

\footnotetext{
2 "The study of second language speech production has benefited from research in several subfields of linguistics. Insights from phonology, acoustic phonetics, and articulatory phonetics have all influenced our understanding of how speakers learn to produce a foreign language."
} 
Para Davidson (2011), nos próximos anos vamos ter um número maior de estudos que incorporam as ferramentas de análise da fonética articulatória (ex.: ultrassom, articulografia eletromagnética (EMA). À medida que novas tecnologias são disponibilizadas aos pesquisadores, novos desafios são lançados e nossas perguntas de pesquisa e escolhas metodológicas precisam ser reformuladas para que novos insights sobre a aprendizagem da fala em L2 sejam gerados.

\section{REFERÊNCIAS}

ALVES, U. K. O papel da instrução explícita na aquisição fonológica do inglês como L2: evidências fornecidas pela Teoria da Otimidade. 2004. 335 f. Dissertação (Mestrado em Linguística Aplicada) - Universidade Católica de Pelotas, Pelotas, 2004.

ALVES, U. K. Estudios recientes sobre la adquisición fonético-fonológica de lenguas extranjeras desarollados en Brasil. In: LUCHINI, Pedro Luis; GARCÍA JURADO, María Amalia; ALVES, Ubiratã Kickhöfel. (org.). Fonética y fonología: articulación entre enseñanza e investigación. Mar del Plata, Argentina: Editora da Universidad Nacional de Mar del Plata, 2015, v. 1. p. 98-109.

BAPTISTA, B. O. The acquisition of English vowels by eleven Brazilian-Portuguese

speakers: An acoustic analysis. 1992. 220 f. Tese (Doutorado em Linguística) - University of California at Los Angeles, Califórnia, 1992.

BARATIERI, J. P. Production of $/ / /$ in the English coda by Brazilian EFL learners: An acoustic-articulatory analysis. $2006.175 \mathrm{f}$. Dissertação (Mestrado em Inglês) - Centro de Comunicação e Expressão, Universidade Federal de Santa Catarina, Florianópolis, 2006.

BECKMAN, M.; KINGSTON, J. Introduction. In: KINGSTON, J.; BECKMAN, M. Papers in laboratory phonology I: between the grammar and physics of speech. Cambridge: Cambridge University Press, 1990. p. 1-16.

BEST, C. A direct realist view of cross-language speech perception. In: STRANGE, W. Speech perception and linguistic experience: issues in cross-language research. Timonuim, MD: York Press, 1995. p. 171-204.

BEST, C.; TYLER, M. D. Nonnative and second-language speech perception: commonalities and complementarities. In: BOHN, O. S.; MUNRO, M. J. language experience in second language speech learning: in honor of James Emil Flege. Amsterdam/Philadelphia: John Benjamins Publishing Company, 2007. p. 13-34.

BETTONI-TECHIO, M. Production of final alveolar stops in Brazilian Portuguese/English interphonology. 2005. 136 f. Dissertação (Mestrado em Inglês) - Centro de Comunicação e Expressão, Universidade Federal de Santa Catarina, Florianópolis, 2005.

BION, R. A. H. The role of listeners' dialect in the perception of foreign-accented vowels. 2007. 102 f. Dissertação (Mestrado em Inglês) - Centro de Comunicação e Expressão, Universidade Federal de Santa Catarina, Florianópolis, 2007. 
CELCE-MURCIA, M. et al. Teaching Pronunciation: A Reference for Teachers of English to Speakers of Other Languages. $2^{\text {nd }}$. ed. New York, NY, Cambridge University Press, 2010.

CORDER, P. Idiosyncratic dialects and error analysis. International Review of Applied Linguistics, v. 5, p. 161-170, 1971.

CORNELIAN Jr., D. Brazilian learners' production of/s/ clusters: Phonological structure and environment. 2003. $78 \mathrm{f}$. Dissertação (Mestrado em Inglês) - Centro de Comunicação e Expressão, Universidade Federal de Santa Catarina, Florianópolis, 2003.

CRISTÓFARO SILVA, T. Fonética e Fonologia do Português. São Paulo: Contexto, 2001.

DAVIDSON, L. Phonetic and phonological factors in the second language production of phonemes and phonotactics. Language and Linguistics Compass, v. 5, n. 3, p. 126-139, 2011.

DE BOT, K. Complexity theory and dynamic systems theory: same or different? In: ORTEGA, Lourdes; HAN, Zhao. Complexity theory and language development: in celebration of Diane Larsen-Freeman. Amsterdam/Philadelphia: John Benjamins Publishers, 2017. p. 51-58.

DELATORRE, F. Brazilian EFL learners' production of vowel epenthesis in words ending in -ed. 2006. 200 f. Dissertação (Mestrado em Inglês) - Centro de Comunicação e Expressão, Universidade Federal de Santa Catarina, Florianópolis, 2006.

DELATORRE, F. Intelligibility of English verbs ending in -ed for Brazilian learners of English listeners. 2017. $312 \mathrm{f}$. Tese (Doutorado em Inglês: Estudos Linguísticos e Literários) - Centro de Comunicação e Expressão, Universidade Federal de Santa Catarina, 2017.

ECKMAN, F. Markedness and the contrastive analysis hypothesis. Language Learning, v. 27, p. 315-330, 1977.

FLEGE, J. E. Second language speech learning: theory, findings, and problems. In: STRANGE, W. Speech Perception and Linguistic Experience: Issues in Cross-Language Research. Timonium, MD: York Press, 1995. p. 233-277.

GASS, S.; SELINKER, L. Second language acquisition: An Introductory Course, $3^{\text {rd }}$ ed. New York: Routledge/Taylor Francis. 2008.

GONÇALVES, A. R. The orthographic signature in second language speech acquisition and processing. 2017. $156 \mathrm{f}$. Tese (Doutorado em Inglês: Estudos Linguísticos e Literários) - Centro de Comunicação e Expressão, Universidade Federal de Santa Catarina, 2017.

KOERICH, R. D. Perception and production of word-final vowel epenthesis by Brazilian EFL students. 2002. $261 \mathrm{f}$. Tese (Doutorado em Inglês: Estudos Linguísticos e Literários) - Centro de Comunicação e Expressão, Universidade Federal de Santa Catarina, 2002.

KLUGE, D. C. Perception and production of English syllable-final nasals by Brazilian learners. 2004. 175 f. Dissertação (Mestrado em Inglês) - Centro de Comunicação e Expressão, Universidade Federal de Santa Catarina, Florianópolis, 2004. 
KLUGE, D. C. Brazilian EFL learners' identification of word-final/m-n/: native/nonnative realizations and effect of visual cues. 2009. 179 f. Tese (Doutorado em Inglês: Estudos Linguísticos e Literários) - Centro de Comunicação e Expressão, Universidade Federal de Santa Catarina, 2009.

KUHL, P. K. Human adults and human infants show a "perceptual magnet effect" for the prototypes of speech categories, monkeys do not. Perception \& Psychophysics, v. 50, n. 2, p. 93-107, 1991.

LADO, R. Linguistics across cultures: Applied linguistics for language teachers. Ann Arbor: University of Michigan Press, 1957.

LARSEN-FREEMAN, D.; CAMERON; L. Complex systems and applied linguistics. Oxford: Oxford University Press, 2008.

LOWIE, W. Lost in state space?: Methodological considerations in Complex Dynamic Theory approaches to second language development research. In: ORTEGA, L.; HAN, Z. Complexity theory and language development: in celebration of Diane LarsenFreeman. Amsterdam/Philadelphia: John Benjamins Publishers, 2017. p. 123-141.

MAJOR, R. C. A model for interlanguage phonology. In: IOUP, G.; WEINBERGER, S. H. Interlanguage phonology: the acquisition of a second language sound system. New York: Newbury House, 1986. p. 101-124.

MITCHELL, R.; MYLES, F. Second language learning theories. 2. ed. London: Hodder Arnold, 2004.

NOBRE-OLIVEIRA, D. The effect of perceptual training on the learning of English vowels by Brazilian Portuguese speakers. 2007.211 f. Tese (Doutorado em Inglês: Estudos Linguísticos e Literários) - Centro de Comunicação e Expressão, Universidade Federal de Santa Catarina, 2007.

PASSARELLA DOS REIS, L. "What do you mean?' Nuclear stress in English as an international language: Uses and interpretations". 2017. 352 f. Tese (Doutorado em Inglês: Estudos Linguísticos e Literários) - Universidade Federal de Santa Catarina, 2017.

PIERREHUMBERT, J.; BECKMAN, M.; LADD, D. R. Conceptual foundations of phonology as a laboratory science. In: BURTONROBERTS, P. C.; DOCHERTY G. Phonological knowledge. Oxford University Press, Oxford, 2000. p. 273-303.

RAUBER, A. S. Perception and production of English vowels by Brazilian EFL speakers. 2006. 218 f. Tese (Doutorado em Inglês: Estudos Linguísticos e Literários) - Centro de Comunicação e Expressão, Universidade Federal de Santa Catarina, 2006.

REBELLO, J. T. The acquisition of English initial/s/ clusters by Brazilian learners. 1997. 198 f. Dissertação (Mestrado em Inglês) Centro de Comunicação e Expressão, Universidade Federal de Santa Catarina, Florianópolis, 1997.

RIBEIRO, T. S. Acoustic correlates of word-stress production in the connected speech of American English and Brazilian Portuguese speakers. 2006. 63 f. Dissertação (Mestrado em Inglês) - Centro de Comunicação e Expressão, Universidade Federal de Santa Catarina, Florianópolis, 2006. 
SCOBBIE, J. M. Interface and overlap in phonetics and phonology. In: RAMCHAND, G.; REISS, C. The Oxford handbook of linguistic interfaces. Oxford, Oxford Univ. Press, 2007. p. 17-52.

SELINKER, L. Interlanguage. International Review of Applied Linguistics, v. 10, p. 209-231, 1972.

SILVA FILHO, J. The production of English syllable-final consonants by Brazilian learners.1998. 123 f. Dissertação (Mestrado em Inglês) - Centro de Comunicação e Expressão, Universidade Federal de Santa Catarina, Florianópolis, 1998.

SILVEIRA, R. The influence of instruction on the perception and production of English word-final consonants. $2004.271 \mathrm{f}$. Tese (Doutorado em Inglês) - Centro de Comunicação e Expressão, Universidade Federal de Santa Catarina, Florianópolis, 2004.

SILVEIRA, R. et al. Percepção, produção e inteligibilidade do inglês falado por usuários brasileiros. In: TOMITCH, L. M. B.; HEBERLE, V. M. (org.). Perspectivas atuais de aprendizagem e ensino de línguas. Florianópolis: LLE/CCE/UFSC, 2017. p. $237-283$.

ZIMMER, M. C.; SILVEIRA, R.; ALVES, U. K. Pronunciation instruction for Brazilians: bringing theory and practice together. Newcastle upon Tyne: Cambridge Scholars Publishing (CSP), 2009.

\section{(ㄷ) (1) $(9$}

